\title{
Reconstructing Teeth with Bite Information
}

\author{
Katrine Hommelhoff Jensen and Jon Sporring \\ Department of Computer Science, University of Copenhagen, Denmark
}

\{katrine, sporring\}@diku.dk

\begin{abstract}
We propose a method for restoring the surface of a tooth crown so that the pose and anatomical features of the tooth will work well for chewing. The system of teeth has been modeled with a 3D statistical multi-object shape model build from 3D scans of dental cast models. The restoration is carried out using the shape model statistics in a Bayesian framework to calculate the most probable tooth crown shape(s), given the fragments of one or more neighboring and opposing tooth crowns. The modeling of and reconstruction with the multi-object shape model has been realized by extending the model with a concept of elasticity that generalizes better to new teeth. The elasticity has been calculated from the surface curvature relations within and between each tooth sample, simulating a prior knowledge of the shape variation.
\end{abstract}

\section{Tooth Reconstruction}

In the dental industry, the design and construction of restorations to be inserted in a patient's mouth is carried out by dental technicians, that are highly trained experts in tooth anatomy and the function of the bite. The task can be to model the missing part of a broken tooth crown, model the crown of a whole missing tooth or even several missing teeth. The restorations are traditionally constructed directly from the materials by hand, but the use of software to model the construction elements of a restoration has been growing rapidly the last couple of years $12 \sqrt{2}$. Other than saving money on the temporary building materials, the software solution saves time, as some of the traditional production steps can be skipped and proper customized 3D modeling tools and automatic routines can speed up the construction work. The existing dental software systems combines the software with a scanning device to produce a 3D surface model of the patients remaining teeth, on which the restoration is to be designed. The modeled restoration can then be exported as a 3D surface model and milled or printed directly in the final material.

One of the most challenging steps towards an automation of dental restoration modeling software is the anatomical deformations of the tooth crowns to be reconstructed. An anatomical correct deformation of a tooth crown surface cannot be calculated exclusively from the size and location of the surrounding surfaces of the scanned data. Some prior knowledge must be added to the system, which describes the shapes and legal deformations of the teeth. It is our goal to develop a system that can learn and describe the complex shape system of the bite, and 
with this knowledge reconstruct the surfaces of missing tooth crown parts, whole teeth, or several teeth from information extracted from scanned data. The work reported in this article is based on [4, and the plaster casts have been scanned by the 3Shape laser scanner, some shown in Figure 1

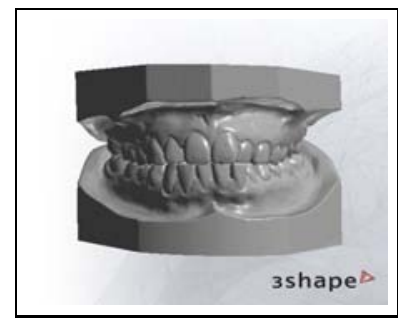

(a)

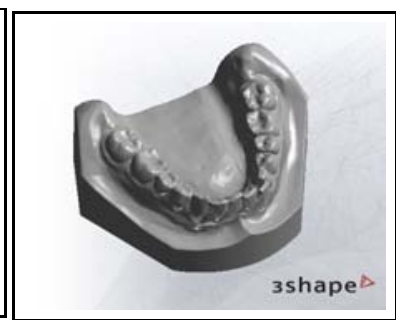

(b)

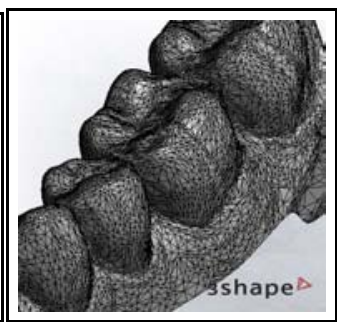

(c)

Fig. 1. Cast models scanned by the 3Shape laser scanner, aligned (a) and the lower jaw (b), and a triangulated surface mesh of a scanned cast model (c). Notice on (c) that the mesh has been decimated to a much lower solution than the usual quality.

\section{$2 \quad$ Shape Modeling}

We operate with the notion of shape as that which is left, when translation, rotation, and scaling is removed. To reconstruct the shape of an incomplete object, we need a model description of the object shape and variability. The classical approach is based on representing and modeling shape as a set of landmarks (see 5678 and references herein). On each training shape, a finite number of landmarks are located on surface features that corresponds between the shapes. This representation is directly applicable in the Active Shape Model (ASM). The biggest disadvantage is the time-consuming manual labor needed. Alternative approaches, that carry a smooth surface implicitly in the shape description, are the Level-set function representation [9] and the Medial representation (M-rep) [10. However, both representations are problematic, since they cannot robustly handle non-closed shape surfaces such as scans of plaster casts of teeth.

Shape Warping has also been studied in the literature: In [11, each training shape landmark is warped to a template shape, where a template shape mesh is projected onto the shape before warping landmarks and mesh vertices back. Based on this idea, 12 introduced a 3D morphable model that use 3D meshes as training data rather than images. Each mesh vertex achieves the same status as a landmark, and the correspondence between the training meshes and the template mesh is estimated from a sparse set of correspondence points, manually marked by the user on each training mesh. In [3] the reconstructions were adjusted for neighboring teeth using local mathematical morphology. The quality of the warping depends on both the complexity of the surfaces and the unknown variation of the samples. Further, when only extracting data from the 
occlusal, frontal, and back sides of the teeth, a significant amount of surface is left unknown. We are thus lead to the idea of keeping the surface representation separated from the data. Further, the reconstruction situations depends on the amount of a tooth that must be reconstructed. If the areas of reconstruction are somehow marked in the process, a better approach is to let the surface conform to the reconstructed data, while respecting the borders between the reconstructed parts and the original data. The task is to create a mesh that can be guided through the landmarks, while keeping the surface smooth and respecting the local and global constraints. This coupling we implement using Variational Implicit Surfaces [13] interpolated between landmarks, and thus we obtain a flexible method, that may be extended with additional information about the anatomical features, and allow us to focus on landmarks only.

A shape will be described as a $3 n$ dimensioned vector of point coordinates

$$
\boldsymbol{x}=\left[\boldsymbol{p}_{1}^{T}, \boldsymbol{p}_{2}^{T}, \ldots, \boldsymbol{p}_{n}^{T}\right]^{T}=\left[x_{1}, y_{1}, z_{1}, x_{2}, y_{2}, z_{2}, \ldots, x_{n}, y_{n}, z_{n}\right]^{T}
$$

with each point representing the position of a landmark and $n$ is the number of landmarks in the shape. Following [8, we use Procrustes analysis to align each shape $\boldsymbol{x}$ to the mean shape $\overline{\boldsymbol{x}}$, where $\overline{\boldsymbol{x}}=\frac{1}{N} \sum_{i=1}^{N} \boldsymbol{x}_{i}$, and $N$ is the number of training samples. To align shapes, we first normalize for translation and size, and then normalize orientation by minimizing the Procrustes distance,

$$
d_{\text {Procrustes }}^{2}=\|\boldsymbol{x}-\overline{\boldsymbol{x}}\|^{2}
$$

We will misuse notation and use $\boldsymbol{x}$ for the normalized shape coordinates in the following. After having aligned the shapes we write,

$$
\mathbf{X}=\left[\left(\boldsymbol{x}_{1}-\overline{\boldsymbol{x}}\right),\left(\boldsymbol{x}_{2}-\overline{\boldsymbol{x}}\right), \ldots,\left(\boldsymbol{x}_{N}-\overline{\boldsymbol{x}}\right)\right]
$$

Then we compute the covariance matrix as

$$
\mathbf{C}=\frac{1}{N} \mathbf{X X}^{T}
$$

and its eigenvalues $\lambda_{i}$ and eigenvectors $\phi_{i}$,

$$
\mathrm{C} \Phi=\Lambda \Phi
$$

where $\boldsymbol{\Lambda}$ is the diagonal matrix of eigenvalues and the columns of the matrix $\boldsymbol{\Phi}$ contains the corresponding eigenvectors or modes. Thus any shape $\boldsymbol{x}$ from the training set can be reproduced by a linear computation of the mean and the principal components as

$$
\boldsymbol{x}=\overline{\boldsymbol{x}}+\boldsymbol{\Phi} \boldsymbol{b}
$$

where $\boldsymbol{b}$ is the vector of shape model parameters. The strength of the model is that the eigenvectors corresponding to the largest eigenvalues model the training set with an error equal to the sum of the neglected eigenvalues.

Our training set for each tooth consist of 12 samples. The landmarks were set by a non-expert with expert assistance and are primarily anatomical, with the 
exception of some pseudo-landmarks. 11 principal components were calculated for each shape model. The relative small training set size could potentially introduce problems regarding the generality, if the dependence on the model statistics is not relaxed in the reconstruction procedure. We will attempt to add artificial eigenmodes to the models, to improve flexibility without hazarding the object shape or overruling the existing eigenmodes. The rationale is that landmarks on the same side of a surface are expected to be correlated proportional with their distance. Thus, if one landmark were to be moved, then we expect that the neighboring landmarks will be effected. We will refer to this as elasticity, which will be described in the following.

\section{$3 \quad$ Model Elasticity}

The modes of variations were calculated from the covariance matrix of the combined data samples. Consider the general $3 n \times 3 n$ covariance matrix with covariances $c_{i j}$

$$
\mathbf{C}=\left[\begin{array}{cccc}
c_{11} & c_{12} & \ldots & c_{13 n} \\
c_{21} & c_{22} & \ldots & c_{23 n} \\
\vdots & \vdots & \ddots & \vdots \\
c_{3 n 1} & c_{3 n 2} & \ldots & c_{3 n 3 n}
\end{array}\right]
$$

If the covariance matrix of the data sample matrix was replaced by the identity covariance matrix $\mathbf{C}_{I d}=\mathbf{I}_{3 n}$, then all landmark coordinates would be independent of each other. This means that combinations of the resulting eigenmodes could move the landmarks of a modeled shape in any direction. In that sense, $\mathbf{C}_{I d}$ defines an under-constrained, lower limit to the shape models. In [14] it has been shown how to add smoothness constrained deformations to a shape model by increasing the correlation between neighboring points in $2 \mathrm{D}$ shapes. The idea is, that when adding a small value to neighboring points in $\mathbf{C}_{I d}$, a covariation between the points is artificially created. Visually, moving a point in the shape will have an elastic effect on the neighbors. The effect of moving a point in a $2 \mathrm{D}$ shape with $\mathbf{C}_{I d}$ and $\mathbf{C}_{I d}$ augmented with with a positive value, e.g. 0.5 , in the covariances between neighboring points, is illustrated in Figure 2.

The actual smoothness used in [14] was, however, not controlled in the relation to the model statistics, they were implemented to substitute model statistics. We need to control the amount of smoothness so that its function is a deformation supplement. Furthermore, we need to re-think the concept of neighbors in 3D, so that the elastic deformation added makes sense and respect the object shape.

In order to relax the tooth shape models we add a small value to all the neighbor-landmark covariances in the covariance matrix. Defining 'neighborhood' is a little more difficult in 3D, though. Neighborhood should be defined more in terms of distance than a number of closest neighbors, and should furthermore be measured over the surface and not necessarily as the shortest distance between two landmarks. As the solid objects teeth are, the smaller artifacts on 

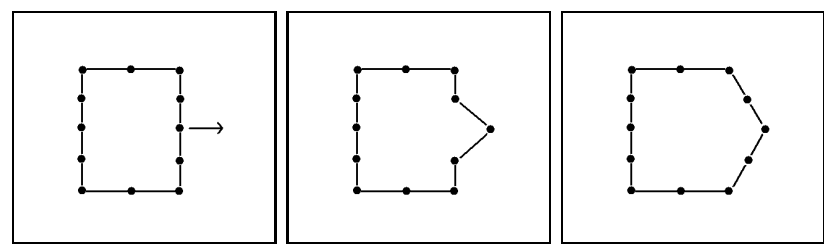

Fig. 2. The original 2D shape (left), the effect on the neighbors when moving a point with $\mathbf{C}_{I d}$ as covariance matrix (middle) and with $\mathbf{C}_{I d}$ augmented with a small value in the covariances of neighboring points (right)

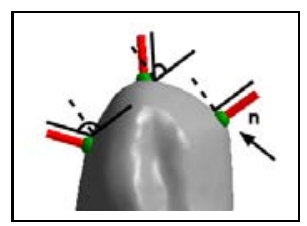

Fig. 3. Three points on a tooth (green) together with their surface normal (red), and angle difference to the rightmost normal. The neighbors to the rightmost landmark is a weighted sum of the surface-geodesic distance and the angle differences.

one side of a given tooth type doesn't have any effect on the smaller surface variations on the other side, disregarding the distance from landmarks on one side to landmarks on the other. In fact, when teeth have more unusual artifact's on the surface and is thus a difficult subject for an over-constrained shape model, it is usually due to abnormal chewing or smaller damages, both of which only have a local effect. The neighborhood of a landmark $\boldsymbol{p}$ could be measured as the landmarks within some fixed distance from $\boldsymbol{p}$. This, however, leaves us with the problem of deciding such a distance. Furthermore, it could introduce some problems regarding the scale of the individual tooth samples - we must choose a method that determines a well defined neighborhood of all shape samples in a model. Let $d_{a b}$ be the mean surface-geodesic distance between landmark $\boldsymbol{p}_{a}$ and $\boldsymbol{p}_{b}$ over the training data. Then, a general way of calculating how much they should affect each other is to calculate this as a weight $0<w_{a b}<1$, where it is our experience that the following function is useful,

$$
w_{a b}=\left(3^{1-\frac{\beta d_{a b}}{d_{\max }}}-1\right) \frac{1+\boldsymbol{n}_{a} \cdot \boldsymbol{n}_{b}}{2}
$$

and where $\boldsymbol{n}_{a}$ are the normal at point $\boldsymbol{p}_{a}$ as shown in Figure [3, $d_{\max }$ is the maximum surface-geodesic distance between points on a shape over the training set, and $\beta$ is a locality parameter typically 2 or 3 . For a given pair of hypothetical landmarks $\boldsymbol{p}_{a}$ and $\boldsymbol{p}_{b}$, where $a \neq b$, we modify the 9 corresponding entries of the covariance matrix, $\mathbf{C}_{\text {elastic }}=\left\{c_{i j}^{\text {elastic }}\right\}$, as follows

$$
c_{i j}^{\text {elastic }}=c_{i j}+w_{a b} \alpha
$$


Table 1. Leave-one-out experiments without and with elasticity of $v=0.2$ and locality $\beta=2$

\begin{tabular}{|l|l|l|}
\hline Shape model & Mean residual error & Mean residual error with elasticity \\
\hline Upper 1st molar & 0.010820445605 & 0.010042073205 \\
Upper 2nd premolar & 0.017800014466 & 0.016395261511 \\
Upper 1st premolar & 0.015877468511 & 0.014705151320 \\
Upper canine & 0.024195164442 & 0.022415077314 \\
Lower 1st molar & 0.011680148542 & 0.010770596564 \\
\hline
\end{tabular}

where $\alpha=\frac{v N}{v_{\text {reg }} n}$ being a parameter to control the amount of regularization, and $v_{\text {reg }}$ is found experimentally such that $v=0$ implies no elasticity added and $v=1$ implies maximum elasticity. The elasticity influences the least significant eigenmodes the most, and should be kept sufficiently small in order not to destroy the statistical properties of the training data.

The parameters will experimentally be found by performing the leave-oneout (LOU) experiments on the corresponding shape models based on PCA of $\mathbf{C}_{\text {elastic. }}$ The goal is thus to find a set of parameters that decreases the residual error for all models in the experiment. The following results were achieved with $v_{\text {reg }}=100$. Table 1 demonstrates the generally better results. With higher values of $v$ than $v=0.2$, the most significant eigenvectors slowly started changing direction, until the corresponding eigenmode changed significance at around $v=$ 0.9 , and therefore we accept $v=0.2$ as the maximum. The amount of non-zero eigenmodes created from $\mathbf{C}_{\text {elastic }}$ are typically as many as $k n$, but a big amount of the least significant eigenmodes can be removed while still keeping the model more general than in the pure statistical model.

\section{Reconstruction with Elasticity}

We now wish to solve the problem of missing data for crown construction. Let $\boldsymbol{y}$ be an incomplete shape vector with $l<n$ points, and $\boldsymbol{x}$ be the corresponding full shape. Then, we wish to find a linear transformation $\mathbf{L}: \mathbb{R}^{n} \mapsto \mathbb{R}^{l}$ such that

$$
\boldsymbol{y}=\mathbf{L} \boldsymbol{x}
$$

This system is an overdetermined system of equations. Since we cannot expect to find a linear combination of the training samples that solves (9) exactly. Instead the values of $\boldsymbol{x}$ can be found by minimizing an energy functional,

$$
E(\boldsymbol{x})=\|\mathbf{L} \boldsymbol{x}-\boldsymbol{y}\|^{2}
$$

which may be solved using the linear least squares method. Assume now that $\boldsymbol{y}$ has been subtracted with the (dimension reduced) mean $\overline{\boldsymbol{x}}$, so that a model approximation can be calculated as $\boldsymbol{x}=\boldsymbol{\Phi} \boldsymbol{b}$. Inserting this into (10) we get

$$
E(\boldsymbol{b})=\|\mathbf{L}(\boldsymbol{\Phi} \boldsymbol{b})-\boldsymbol{y}\|^{2}
$$


Table 2. Reconstruction of molar training sample for the normal model and the elastic model that includes regularized elasticity

\begin{tabular}{|l|l|l|}
\hline Removed landmarks & Normal model & Elastic model \\
out of 44 total & $E_{\mathbf{r}_{r e c}}$ & $E_{\mathbf{r}_{r e c}}$ \\
\hline 1 & 0.00159 & 0.00075 \\
5 & 0.02201 & 0.00676 \\
12 & 0.04915 & 0.01711 \\
36 & 0.65755 & 0.65539 \\
36 distr & 0.19626 & 0.11631 \\
\hline
\end{tabular}

However, a fundamental problem with least square fitting of a model to data is that of overfitting. Basically, there is a lot of uncertainties in the data, and the model is only an approximation to the real physical system. Hence, we formulate the problem in the Bayes setting: Given an incomplete shape vector $\boldsymbol{y}$, the reconstruction problem consist of finding the optimal model coefficients $\boldsymbol{b}$ for $\boldsymbol{y}$. In terms of probability:

$$
P(\boldsymbol{b} \mid \boldsymbol{y})=\frac{P(\boldsymbol{y} \mid \boldsymbol{b}) P(\boldsymbol{b})}{P(\boldsymbol{y})} \propto P(\boldsymbol{y} \mid \boldsymbol{b}) P(\boldsymbol{b})
$$

This states that the optimal coefficients $\boldsymbol{b}$ will be the ones with maximum probability, conditioned to $\boldsymbol{y}$. Both the prior probability $P(\boldsymbol{b})$ and the likelihood $P(\boldsymbol{y} \mid \boldsymbol{b})$ can be derived from the shape model definition. We use a normally distributed on $\boldsymbol{b}$ with a zero mean and covariance matrix equal to the identity, $\boldsymbol{b} \sim N(0, \mathbf{I})$, the probability density can then be written as:

$$
P(\boldsymbol{b})=(2 \pi)^{-m / 2} \exp \left(-\frac{\|\boldsymbol{b}\|^{2}}{2}\right)
$$

and the probability density of the likelihood

$$
P(\boldsymbol{y} \mid \boldsymbol{b})=\left(2 \pi \sigma^{2}\right)^{-l / 2} \exp \left(-\frac{\|\left(\mathbf{L}(\boldsymbol{\Phi} \boldsymbol{b})-\boldsymbol{y} \|^{2}\right.}{2 \sigma^{2}}\right)
$$

The point of maximum posteriori is found to be 15

$$
\boldsymbol{x}=\overline{\boldsymbol{x}}+\boldsymbol{\Phi} \mathbf{V} \operatorname{diag}\left(\frac{w_{i}}{w_{i}^{2}+\sigma^{2}}\right) \mathbf{U}^{T}(\boldsymbol{y}-\mathbf{L} \overline{\boldsymbol{x}})
$$

The reconstruction error for various missing landmarks is shown in Table 2. The reduced elastic model results in a significantly better reconstruction, compared to the normal covariance reconstruction.

The reconstruction with the elastic models showed that some kind of regularization is necessary, due to the increased number of eigenmodes and the effect of the neighborhood relations on the reconstruction. As the complexity of the reconstruction problem increases, the resulting shape quickly becomes very distorted. Our experiments indicate that only a small number of artificial 

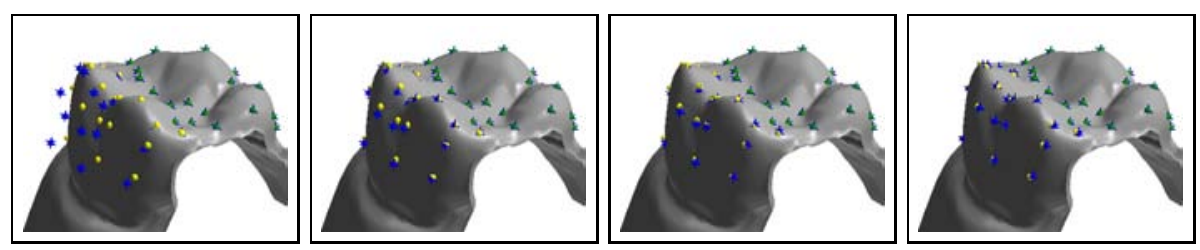

Fig. 4. Reconstruction molar training sample with elastic model, and different amount of elastic eigenmodes removed. Three sets of landmarks are shown: origional (green), the reconstructed (blue), and the ground truth (yellow). From left: 0\%, $50 \%, 60 \%$ and $80 \%$ eigenmodes removed.

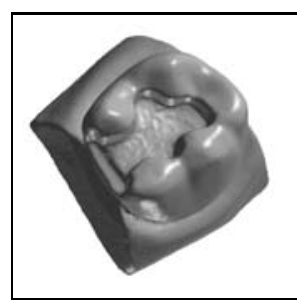

(a)

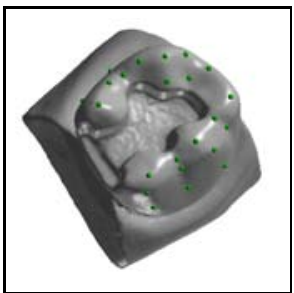

(b)

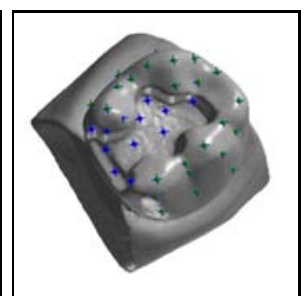

(c)

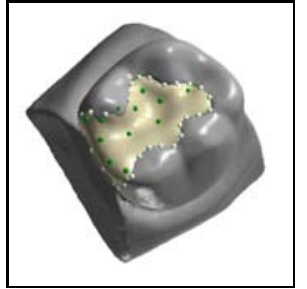

(d)

Fig. 5. Reconstruction molar from elastic shape model, with elasticity $v=0.1$ and locality parameter $\beta=3$. (a) Original tooth with surface part to be reconstructed. (b) Landmarks to reconstruct from. (c) Reconstructed landmarks (blue). (d) Reconstruction with surface mesh.

eigenmodes should be used for optimal reconstruction. This is show in Figure 4 . With this approach we are now able to reconstruct hitherto unseen teeth such as shown in Figure 5

\section{Bite Constrained Reconstruction}

One of the limitations of a PDM-based shape model is the undefined limit of shape variation in each eigenmode. We assume limits of three standard deviations of the mean, assuming a Gaussian data distribution. In PCA, when fitting the data to an affine subspace, we cannot guarantee the displacement vectors in some eigenmode not to overlap. The approximated limits on the modes of variation makes it a common problem that the PDM produces shapes with illegal borderoverlaps. This is a problem of particular importance when modeling multiple tooth shapes with one model, since the surfaces of neighboring and antagonist teeth in a natural bite are not only close, they also share one or more contact points, and thus inter-model border overlapping is very likely.

In the reconstruction, we wish to maximize the posterior probability of the model parameters $\boldsymbol{b}$ given the incomplete shape vector $\boldsymbol{y}$, by minimizing $\|(\mathbf{L} \boldsymbol{\Phi}) \boldsymbol{b}-\boldsymbol{y}\|^{2}$. Let $\boldsymbol{p}_{y}$ be the landmark in $\boldsymbol{y}$ that was just given a new position away from the collision, and let $\boldsymbol{p}_{m}$ be the corresponding landmark in the 


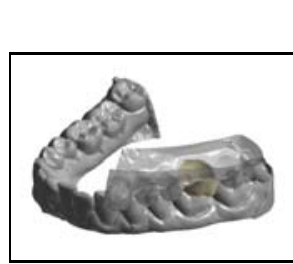

(a)

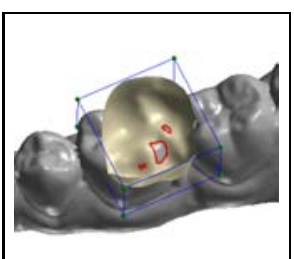

(b)

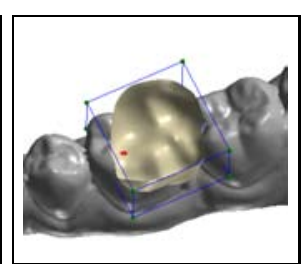

(c)

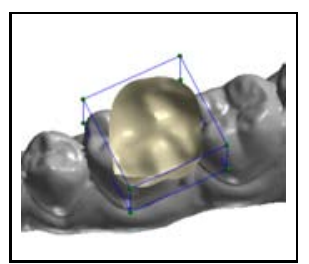

(d)

Fig. 6. Reconstruction of upper molar with collision with antagonist. Iterations in the collision response algorithm. (a) Overview. (b)-(d) iterative reduction in collisions.

model to approximate $\boldsymbol{p}_{y}$. Let Let $\boldsymbol{n}$ be a normal vector in the direction of the penetration. Then, by minimizing the distance between the landmarks as

$$
\left\|\boldsymbol{n}^{T} \boldsymbol{p}_{m}-\boldsymbol{n}^{T} \boldsymbol{p}_{y}\right\|^{2}
$$

the distance between the landmarks is only measured in the direction of $\boldsymbol{n}$. This expression increases the possibility of estimating a $\boldsymbol{p}_{m}$ in a non-collision position, by relaxing the movement of $\boldsymbol{p}_{m}$ in the plane through $\boldsymbol{p}_{y}$ with the normal $\boldsymbol{n}$, thus motivating the most probably $\boldsymbol{p}_{m}$ close to this plane. We will also refer to this as a plane constraint. To respond to collisions, we apply an iterative algorithm, where we in each step, seek the landmark of deepest penetration. This landmark is then pushed out along the surface normal and apply the plane constraint, i.e. require, that this landmark no longer can move in the normal direction. Steps from this algorithm is illustrated in Figure 6 .

\section{Conclusion}

We have presented a system for reconstructing teeth based on an extension of the Principal Component Analysis. Our extensions include both an elasticity term for the covariance matrix and collision avoidance for antagonist teeth. The conclusion is, that the reconstruction generalize well in terms of missing data, collisions are minimized for improved biting, and preliminary clinical evaluation indicate that the resulting models visualized by variational implicit surfaces are more natural looking that standard reconstructions.

\section{References}

1. Modgil, S., Hutton, T., Hammond, P., Davenport, J.: Combining biometric and symbolic models for customised, automated prosthesis design. Artificial Intelligence in Medicine 25, 227-245 (2002)

2. Gürke, S.: Restoration of teeth by geometrically deformable models, http://citeseer.comp.nus.edu.sg/gurke97restoration.html (1997)

3. Hayashi, T., Tsuchida, J., Kato, K.: Semi-automatic design of tooth crown using a 3-D dental CAD system, Vocs-1B. In: Proceedings of the 22nd Annual EMBS International Conferenc, Chicago IL, USA,pp. 565-566 (July 2000) 
4. Jensen, K.H.: A constrained 3D statistical shape model for automatic reconstruction of teeth in a human bite. Master's thesis, University of Copenhagen (October 2006) ftp://ftp.diku.dk/diku/image/publications/jensen.061023.pdf

5. Dryden, I., Mardia, K.: Statistical Shape Analysis. John Wiley \& Sons, New York (1998)

6. Cootes, T., Taylor, C., Cooper, D.H., Graham, J.: Active shape models - their training and application. Computer Vision and Image Understanding 61(1) (1995)

7. Cootes, T., Taylor, C.: Statistical models of appearance for computer vision. Technical report, University of Manchester (March 2004)

8. Bookstein, F.L.: Shape and the information in medical images: A decade of morphometric synthesis. Computer Vision and Image Understanding 66(2), 97-118 (1997)

9. Sethian, J.A.: Level Set Methods and Fast Marching Methods. Cambridge University Press, Cambridge (1999)

10. Pizer, S., Thall, A., Chen, D.: M-reps: A new object representation for graphics. Technical report, University of North Carolina (1999)

11. Hutton, T.J., Buxton, B.F., Hammond, P.: Dense surface point distribution models of the human face. IEEE Workshop on Mathematical Methods in Biomedical Image Analysis (MMBIA),153 (2001)

12. Blanz, V., Vetter, T.: A morphable model for the synthesis of 3d faces. In: Proc. of SIGGRAPH '99, Los Angeles,pp. 187-194 (August 1999)

13. Turk, G., O'Brien, J.F.: Variational implicit surfaces. Technical report, Georgia Institute of Technology (May 1999) Tech Report GIT-GVU-99-15

14. Wang, Y., Staib, L.H.: Boundary finding with correspondence using statistical shape models. In: Proc. IEEE Conf. Computer Vision and Pattern Recognition, pp.338 - 345 (1998)

15. Blanz, V., Vetter, T.: Reconstructing the complete $3 \mathrm{~d}$ shape of faces from partial information. Technical report, University of Freiburg (2001) Computer Graphics Technical Report No. 1 